\title{
24-Hour urinary glucose excretion in assessment of control in juvenile diabetes mellitus
}

\author{
R. J. LEVINSKY, R. S. TROMPETER, and D. B. GRANT \\ From the Institute of Child Health and the Hospital for Sick Children, London
}

\begin{abstract}
Levinsky, R. J., Trompeter, R. S., and Grant, D. B. (1976). Archives of Disease in Childhood, 51, 463. 24-Hour urinary glucose excretion in assessment of control in juvenile diabetes mellitus. 24-Hour urinary glucose excretion was measured in 43 juvenile diabetics during treatment as outpatients. In 20 children studied twice over 1-3 months there was good correlation between glucose excretion on each occasion. Subdivision of the collections into the periods $08 \cdot 00-20 \cdot 00$ and $20 \cdot 00-08 \cdot 00$ hours gave slightly less consistent results with correlation coefficients of 0.83 and $0 \cdot 80$, respectively, between the results of the repeat tests.

In 37 prepubertal children, 24-hour glucose concentration and height velocity over the previous year were compared, and a highly significant negative correlation found. 10 of the 12 children with glucose excretion $>40 \mathrm{~g} / \mathrm{d}$ had height velocities more than 1 SD below the mean for age, while only 2 of the 25 subjects excreting $<40 \mathrm{~g} / \mathrm{d}$ had height velocities more than $1 \mathrm{SD}$ below the mean.

The results indicate that estimation of 24-hour urine glucose excretion can be a useful index for monitoring treatment and that subdivision of the total collection may be of value in selecting the most suitable insulin regimen for the patient.
\end{abstract}

There is still controversy as to what constitutes good control in juvenile diabetes and widely different criteria for assessing treatment are used in different clinics. While frequent blood glucose estimations are used in some centres to regulate treatment (Baker, Blumenthal, and Kaye, 1972), in many clinics estimation of glycosuria at different times of the day provides the yardstick for controlling insulin dosage and diet. Estimation of 24-hour urine glucose excretion has also been advocated as a means of assessing treatment (Larsson and Ström, 1956; White, 1965; Weil, 1968) but does not seem to have gained wide acceptance by paediatricians. However, Forman, Goldstein, and Genal (1974) have described encouraging results using 24-hour urine glucose excretion, subdivided into three 4-hour collections and a 12-hour overnight collection as a guide to the choice of insulin dose and timing.

The present study was carried out to determine whether the pattern of glucose excretion was relatively constant in individual patients, and to try to assess whether estimation of 24-hour urine glucose

Received 22 September 1975. excretion could provide a useful index of diabetic control.

\section{Subjects}

Twenty-five boys and 18 girls aged 5 to 14 years, who had been diabetic for 2 to 11 years, were investigated as outpatients. All were clinically well at the time and were receiving insulin injections once or twice daily with a carbohydrate exchange diet. Detailed dietary histories in 20 children indicated that approximately $40 \%$ of the daily calorie intake was derived from carbohydrate (range $35-43 \%$ ). Insulin dosage was regulated by twice or three times daily testing of doublevoided urine samples at home, using Clinitest tablets.

\section{Methods}

24-hour specimens of urine, subdivided into collections between 8.00-20.00 hours and 20.00-08.00 hours, were analysed for glucose concentration using the $o$-toluidine method (Cooper and McDaniel, 1970). Specimens were collected into bottles containing methiolate during the weekend and brought to the clinic 2 days later. In 20 children the test was repeated 1-3 months later after a period in which there had been no significant change in diet or insulin dosage. 
Growth rates were calculated from clinic measurements taken over the previous year and have been expressed as the standard deviation score (SDS) for height velocity (Tanner et al., 1971). Boys 13 years and over, and girls 11 years and over, were excluded from this analysis as variation in the timing of puberty precluded the use of SDS in the older children. Linear regression analysis was used to assess the significance of results.

\section{Results}

The results of the paired tests of 24-hour urine glucose excretion, separated by intervals of 1-3 months, in 20 children are shown in Fig. 1. A

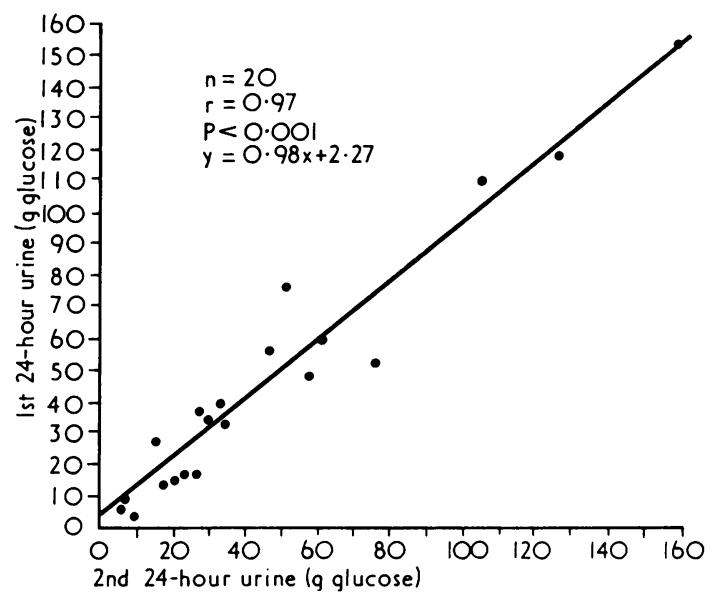

Fig. 1.-Correlation between paired 24-hour urine glucose collections in 20 subjects.

close correlation for the results of the two tests was obtained $(r=0.97, P<0.001)$. The results of the 12-hour subcollections are shown in Fig. 2, which compares the $08 \cdot 00-20 \cdot 00$ hour and $20 \cdot 00$ $08 \cdot 00$ hour urine glucose excretion during the two tests. Significant correlation was found between the $08 \cdot 00-20 \cdot 00$ and $20 \cdot 00-08 \cdot 00$ hour urine glucose excretion $(r=0.65, P<0.001)$. There was also good correlation between the paired results for the 8.00-20.00 hour collections $(r=0.83$, $P<0.001$ ) and the $20 \cdot 00-8 \cdot 00$ hour collections $(r=0 \cdot 80, P<0 \cdot 001)$.

The relation between 24-hour glucose excretion and height velocity in 37 prepubertal children is shown in Fig. 3. A highly significant negative correlation between 24-hour urine glucose excretion and height velocity was obtained $(r=-0.63$, $P<0.001)$. All but 2 of the 25 children excreting $<40 \mathrm{~g}$ glucose each day had height velocties above -1 SDS, while only 2 of the 12 children with urine glucose excretion $>40 \mathrm{~g} /$ day had height

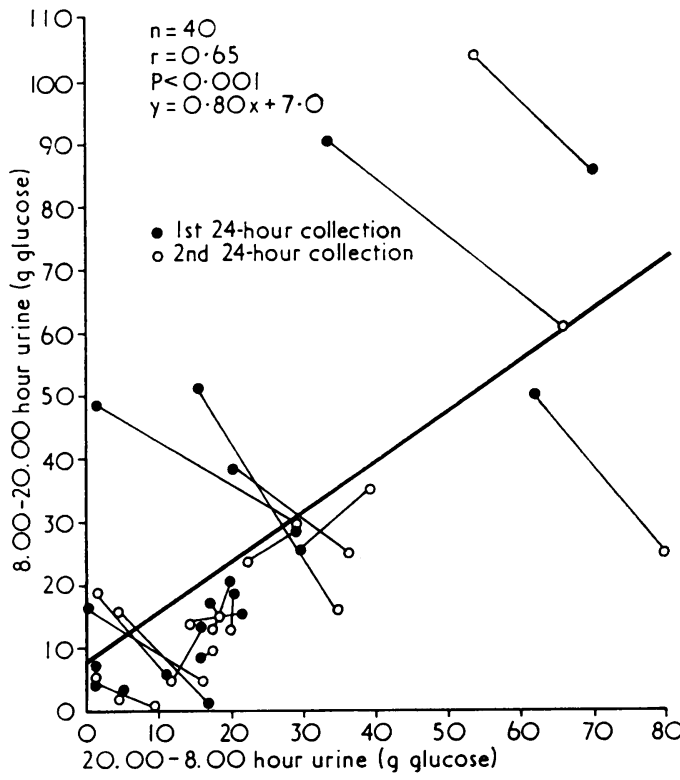

Fig. 2.-Correlation between 8.00-20.00 and 20.00-8.00 hour urine glucose collections. Joined lines indicate paired collections 1-3 months apart in same subject.

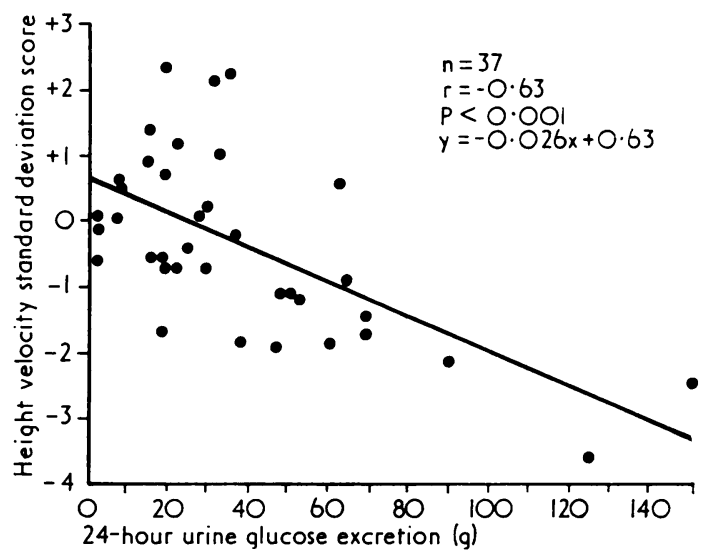

FIG. 3.-Correlation between height velocity expressed as standard deviation score and 24-hour urine glucose excretion in 37 prepubertal children.

velocities above -1 SDS. A similar correlation was obtained when height velocity was compared with urine glucose excretion expressed as a percentage of carbohydrate intake $(r=-0.62, P$ $<0.001)$.

Urine glucose concentration and urine volume were compared in 120 12-hour collections. As 
might be expected, a significant correlation between glucose concentration and volume was found $(\mathrm{r}=0.36, \mathrm{P}<0.001)$, but there was considerable scatter of results and urine volume did not reflect glucose concentration in many of the collections.

Analysis of insulin dose (range $0 \cdot 43-1 \cdot 81$ units/ $\mathrm{kg}$ ) against height velocity and 24-hour urine glucose excretion did not show any significant correlation.

\section{Discussion}

Control of treatment still presents problems in many children with diabetes. As insulin injections cannot completely mimic the regulated secretion of insulin by the pancreas into the portal circulation, blood glucose levels may vary considerably with activity and food intake, and as a result, random estimation of blood glucose may provide little information as to the efficacy of treatment. Frequent estimates of blood glucose, either in hospital or at home (Baker et al., 1972), may provide a better index of diabetic control, but many paediatricians are reluctant to take multiple blood samples from their younger patients and prefer to base their treatment on the pattern of glycosuria in samples obtained at different times of the day. This may occasionally be misleading as $2 \%$ glucose concentration in a small volume of urine is insignificant glycosuria, whereas $2 \%$ in a large volume may represent a large loss.

The present study indicates that estimation of 24-hour urine glucose excretion may provide a very useful supplement to such tests. For example, in 2 of our patients with delayed growth, who persistently reported little glycosuria when testing their urine at home with Clinitest tablets, the daily glucose excretion ranged from 124-156 g, and we can only conclude that these patients were fabricating their test results. Clearly it would be easy for a patient to 'cheat' with a 24-hour urine collection, but if this was suspected analysis of urinary creatinine could be used to check the completeness of the collection (Barratt, 1974).

The results also indicate that in stable diabetics, urinary glucose excretion is relatively constant from day to day over periods of up to 3 months. The pattern of glucose excretion during the day and night is also fairly stable and we agree with Forman et al. (1974) that estimation of urinary glucose over different periods of the day may be useful in determining the most suitable insulin regimen for each patient. In particular, fractionated collection may be useful in patients who become hypoglycaemic during the night and awaken with rebound hyperglycaemia and ketonuria. In these patients it is tempting to increase the insulin dosage, thereby exacerbating this 'Somogyi' effect (Somogyi, 1959). Forman et al. (1974) used three 4 hour collections followed by a 12-hour overnight collection for glucose analysis and readjusted the type of insulin and diet in the light of the results. We have not explored the possibility of using samples collected for periods of $<12$ hours and we cannot comment on the value of subdividing the 24-hour collection into several shorter periods. Forman et al. (1974) also proposed that once the pattern of glucose excretion had been established for an individual patient, the measurement of urine volume might provide a reliable index of the degree of glycosuria. Our results do not support this view. Though there was a statistically significant relationship between urine volume and glucose concentration, the correlation was not close enough to allow useful assessment of glucose concentration from the urine volume.

The above findings also indicate what might be considered good control in juvenile diabetes. While all the children in the study were clinically well with no evidence of retinopathy or significant proteinuria, a few had delayed growth. Only 2 of the 12 patients excreting more than $40 \mathrm{~g}$ glucose each day had height velocities above -1 SDS. Conversely, 23 of the 25 children with daily glucose excretion less than $40 \mathrm{~g}$ had height velocities above -1 SDS. Despite controversy over whether linear growth is related to the degree of control in juvenile diabetes (Pond, 1970; Jivani and Rayner, 1973), we suggest that glucose excretion in excess of $40 \mathrm{~g} / \mathrm{d}$ should be considered unsatisfactory and believe that treatment should be adjusted to keep the glucose excretion below this level.

Clearly, estimation of the 24-hour urine glucose excretion can only give a rough index of diabetic control as it is dependent on other factors such as renal threshold for glucose. However we agree with others that it provides a useful index of the effectiveness of treatment and believe that it deserves wider application in the management of juvenile diabetes.

\section{REFERENCES}

Baker, L., Blumenthal, A., and Kaye, R. (1972). Monitoring diabetic control in the home, a new approach. Pediatric Research, 6, 396.

Barratt, T. M. (1974). Assessment of renal function in children. Modern Trends in Paediatrics-4, p. 181. Ed. by J. Apley. Butterworths, London.

Cooper, G. R., and McDaniel, N. (1970). The determination of glucose by the ortho-Toluidine method. Standard Methods of Clinical Chemistry, Vol. 6, p. 159. Ed. by R. MacDonald. Academic Press, New York and London.

Forman, B. H., Goldstein, P. S., and Genel, M. (1974). Management of juvenile diabetes mellitus: usefulness of 24 hour fractional quantitative urine glucose. Pediatrics, 53, 257.

Jivani, S. K. M., and Rayner, P. H. W. (1973). Does control influence the growth of diabetic children? Archives of Disease in Childhood, 48, 109. 
Larsson, Y., and Ström, L. (1956). Treatment of juvenile diabetes with particular reference to diet. Annales Paediatrici, 186, 270.

Pond, H. (1970). Some aspects of growth in diabetic children. Postgraduate Medical fournal, 46, Suppl. Sept., 616.

Somogyi, M. (1959). Exacerbation of diabetes by excess insulin action. American fournal of Medicine, 26, 169.

Tanner, J. M., Whitehouse, R. H., Hughes, P. C. R., and Vince, F. P. (1971). Effect of human growth hormone treatment for 1-7 years on growth of 100 children, with growth hormone deficiency, low birthweight, inherited smallness, Turner's syndrome, and other complaints. Archives of Disease in Childhood, 46, 745 .
Weil, W. B., Jr. (1968). Juvenile diabetes mellitus. New England fournal of Medicine, 270, 829.

White, P. (1965). The child with diabetes. Medical Clinics of North America, 49, 1069.

We thank the Department of Chemical Pathology at the Hospital for Sick Children for urine glucose estimatiors.

Correspondence to Dr. D. B. Grant, Hospital for Sick Children, Great Ormond Street, London WC1.

The following articles will appear in future issues of this journal:

Long-term assessment of children exposed in utero to carbimazole. A. M. McCarroll, M. Hutchinson, R. McAuley, and D. A. D. Montgomery.

Idiopathic hypoglycaemia in sibs with morphological evidence of nesidioblastosis of the pancreas. D. Woo, J. W. Scopes, and J. M. Polak.

Achondrogenesis type I: a familial subvariant? I. Lauder, H. A. Ellis, T. Ashcroft, and A. Burridge.

Pre- and postoperative growth in persistent ductus arteriosus. D. Pickering, V. Rose, and B. Armstrong.

Is cystic fibrosis an acid mucopolysaccharidosis? $\quad$ R. N. Mukherji, P. D. Moss, and C. K. Heffernan.

Treatment of dermatomyositis in childhood. V. Dubowitz.

Growth hormone deficiency after treatment of acute leukaemia in children. $S$. M. Shalet, C. G. Beardwell, P. H. Morris Jones, and D. Pearson.

Association of cystic fibrosis with allergy. J. O. Warner, B. W. Taylor, A. P. Norman, and J. F. Soothill. 